\title{
Procesos argumentativos que conforman las reflexiones del profesorado chileno sobre sus experiencias de colaboración en el marco del Diseño Universal para el Aprendizaje
}

Moreno Díaz, Karina del Carmen; Soto González, Rodolfo Israel; Oyarzún Maldonado, Cristian José Procesos argumentativos que conforman las reflexiones del profesorado chileno sobre sus experiencias de colaboración en el marco del Diseño Universal para el Aprendizaje

Revista Educación, vol. 45, núm. 2, 2021

Universidad de Costa Rica, Costa Rica

Disponible en: https://www.redalyc.org/articulo.oa?id=44066178015

DOl: https://doi.org/10.15517/revedu.v45i1.43500

\section{(c) $19 \Theta(\Theta$}

Esta obra está bajo una Licencia Creative Commons Atribución-NoComercial-SinDerivar 3.0 Internacional. 


\section{Procesos argumentativos que conforman las reflexiones del profesorado chileno sobre sus experiencias de colaboración en el marco del Diseño Universal para el Aprendizaje}

Argumentative Processes among Chilean Teachers Regarding their Collaborative experience as part of the Universal Design for Learning Framework

Karina del Carmen Moreno Díaz

Universidad de Barcelona, España

karina.moreno@usach.cl

iD https://orcid.org/0000-0003-0312-735X

\author{
Rodolfo Israel Soto González \\ Universidad de Barcelona, España \\ rodolfo.soto@usach.cl
}

iD https://orcid.org/0000-0003-3061-6036

Cristian José Oyarzún Maldonado

Universidad de Chile, Chile

cristian.oyarzunm@usach.cl

iD https://orcid.org/0000-0003-4811-8633
DOI: https://doi.org/10.15517/revedu.v45i1.43500

Redalyc: https://www.redalyc.org/articulo.oa?

$\mathrm{id}=44066178015$
Recepción: 04 Septiembre 2020

Aprobación: 08 Octubre 2020

\section{ReSUMEN:}

El Diseño Universal para el Aprendizaje [DUA] es un marco de referencia orientado a construir mayores y variadas oportunidades para que todo el alumnado pueda aprender y participar. Su implementación, prescrita por la promulgación del Decreto 83 en Chile, requiere dinámicas de colaboración entre el profesorado. Así, este estudio establece como objetivo comprender las reflexiones del cuerpo docente sobre sus experiencias de colaboración en el marco del DUA. Desde un enfoque cualitativo, se realizaron entrevistas a 12 profesores y profesoras de educación básica, organizadas en dos etapas para representar la complejidad de las reflexiones: episódica y argumentativa. El material fue analizado desde herramientas derivadas de la teoría fundamentada. Los resultados muestran que los profesores y las profesoras reconocen que su actividad colaborativa dirigida a diversificar la enseñanza está condicionada por factores institucionales. Además, las experiencias colaborativas satisfactorias entre agentes educativos se caracterizan por la interdependencia, reconocimiento mutuo y la confianza. Las consecuencias favorables en la motivación y el desarrollo del alumnado se facilitan, de acuerdo con las reflexiones del cuerpo docente, cuando se trabaja la diversificación conjuntamente con el aprendizaje cooperativo. Estos resultados son discutidos a un nivel institucional, de relación profesional y de aula, concluyendo que cada elemento abordado reflexivamente por los profesores y las profesoras es relevante y forman una estructura articulada. Las principales implicancias son: comprender la implementación del DUA como un proceso reflexivo conjunto; crear condiciones culturales, relacionales, formativas, de tiempo y de política educativa, y concretar el DUA en el aula con estructuras de aprendizaje cooperativo.

Palabras Clave: Profesión docente, Diseño Universal para el Aprendizaje, Trabajo colaborativo, Cooperación, Diversidad, Asociación profesional, Actividades escolares, Estrategias de aprendizaje.

\section{Abstract:}

The Universal Design for Learning [UDL] is a series of guidelines that serve as a framework to develop more varied and engaging learning opportunities for students. Chile adopted the UDL after passing Decree 83. Implementation of UDL requires strong collaboration among educators. The objective of this study was to understand teacher collaboration within the UDS based on their reflections. A qualitative research method based on a series of interviews was conducted among twelve primary school teachers. The interviews were divided into two stages - argumentative and episodic - to represent the complexity of the reflections. Responses were analysed based on a grounded theory approach. According to the results, teachers acknowledged 
that any collaborative effort aimed at diversifying teaching was conditioned by institutional factors. Furthermore, successful collaborative experiences are characterized by interdependence, mutual recognition, and trust. Positive results about student motivation and student development are included. Teacher reflection reveals that when diversification is worked together with cooperative learning. These results are discussed at an institutional, professional relationship and classroom level, concluding that each element thoughtfully addressed by teachers is relevant and forms an articulated structure. The main implications include: understanding the implementation of the UDL as a joint reflective process, creating the cultural, relational, training, time and educational policy-related conditions and performing UDL in the classroom using cooperative learning structures.

KEYwoRDs: Teaching Profession, Universal Design for Learning, Collaborative Work, Cooperation, Diversity, Professional Association, School Activities, Learning Strategies.

\section{INTRODUCCIÓN}

Los procesos de globalización han aumentado la diversidad en los sistemas escolares (Villegas, Ciotoli y Lucas, 2017). No es posible comprender esta diversidad mediante simples atributos asignados externamente, sino entendiendo que cada persona es portadora de una voz que entremezcla una multiplicidad de posiciones (Wegerif et al., 2013). Si cada estudiante es una persona singular y única, no es apropiada una respuesta educativa para tipos de estudiantes, sino que es necesario centrarse en lo disponible para todos y todas en la comunidad de aprendizaje (Florian, 2017). Es así como el foco de cambio es el propio contexto del estudiantado, incluyendo la cultura, la organización y las prácticas educativas para atender a la diversidad, siendo esta última la fuente de aprendizaje y crecimiento para todos y todas (Blanco, 2006).

En concreto, la existencia de la diversidad es el punto de partida de cualquier proceso de enseñanza y aprendizaje (Onrubia, 1993) y, por tanto, los currículos inflexibles y de talla única generan barreras para el aprendizaje y discapacitan a algunos miembros del estudiantado (Center for Applied Special Technology, 2011). En contraste, para responder adecuadamente a la diversidad, se necesita construir currículos con una flexibilidad inherente mediante diversos materiales, métodos y medios de evaluación a favor de objetivos claros (Hitchcock, Meyer, Rose y Jackson, 2002). Un marco educativo de referencia para maximizar las oportunidades de todo el estudiantado corresponde al Diseño Universal para el Aprendizaje [DUA] (Meyer, Rose y Gordon, 2014), siendo un enfoque que promueve el diseño de entornos de aprendizaje flexibles y de contenido accesible (Al-Azawei, Serenelli y Lundqvist, 2016). En Chile se ha ido aplicando el DUA de forma gradual en las aulas de nivel educativo parvulario y básico, esto a partir de la promulgación en 2015 del Decreto 83 Diversificación de la Enseñanza. En este Decreto se aprueban criterios y orientaciones de adecuación curricular y diversificación de la enseñanza en el marco del DUA.

La introducción del DUA en las aulas conlleva un cambio de perspectiva importante para el grupo de profesionales de la educación con respecto a la colaboración, ya que esta última no se comprende como algo que ayuda a la labor docente, sino como un factor central y decisivo (Meyer et al., 2014). Es más, la inclusión educativa, en términos generales, requiere de la colaboración profesional en su puesta en práctica y progreso (Ainscow, 1995; Echeita, 2006). De esta manera, el aislamiento profesional constituye un obstáculo para la promoción de prácticas educativas orientadas a la atención de la diversidad (Sandoval, 2008). Por lo tanto, con objeto de implementar efectivamente el DUA es necesario construir una cultura de colaboración entre el personal docente; participar en una comunidad de aprendizaje que les permita desarrollar sus fortalezas; reflexionar sobre la enseñanza y explorar nuevas formas para abordar la diversidad del estudiantado y, de este modo, construir el entorno de aprendizaje que cada niño, niña y joven merece y necesita para su desarrollo (Meyer et al., 2014).

A pesar del rol decisivo de la colaboración profesional docente en la promoción de procesos inclusivos, los contextos escolares típicamente presentan culturas de fragmentación que dificultan su consolidación (Hugel, 2009). Además, se desprende desde el cúmulo de evidencia investigativa la existencia de múltiples obstáculos a la colaboración entre los integrantes del cuerpo docente, entre las que se encuentran la sobrecarga de trabajo, la estructura de los grupos de clase, la escasez de tiempo, la complejidad de las tareas y la 
competitividad cultural (Gravel, Le Bossé y Fournier, 2019). También, actúa como un obstaculizador el auge de políticas educativas que transforman la docencia en una labor individual orientada hacia tareas estandarizadas (Oyarzún, Soto y Moreno, 2019).

En contraste, cuando dinámicas colaborativas se instalan en la escuela, el personal docente experimenta satisfacción (Knox y Anfara, 2013) y, al participar en múltiples procesos de apoyo social, se favorece el desarrollo profesional en términos de construcción de herramientas (Hauge y Wang, 2019). Es importante precisar que la colaboración asume variadas y particulares formas, dependiendo de distintos factores personales y contextuales que la facilitan, modulan o impiden (Cabezas, Medeiros, Inostroza, Gómez y Loyola, 2017).

Los antecedentes expuestos muestran la necesidad de fomentar instancias colaborativas entre agentes educativos a fin de concretar, en las prácticas educativas, sistemas diversificados de apoyo al alumnado. Asimismo, se constata que este proceso puede enfrentar diferentes problemáticas y, por ende, no puede concebirse como una tarea sencilla de abordar. Ante tal desafiante necesidad, las reflexiones de los propios protagonistas respecto a las sucesivas experiencias profesionales desde el rol docente pueden ofrecer miradas críticas para comprender estos fenómenos. Por consiguiente, el acto de examinar las reflexiones de los profesores y las profesoras sobre estas cuestiones puede dar luces sobre cómo canalizar ajustadamente las dinámicas colaborativas en comunidades docentes a favor de la participación de todo el estudiantado, independientemente de las características de estos últimos. De este modo, el presente artículo tiene por objetivo comprender las reflexiones que profesores y profesoras de enseñanza básica construyen sobre sus experiencias de colaboración en el marco de la gradual implementación del DUA en Chile por medio de una investigación cualitativa realizada con la participación de 12 docentes.

\section{ANTECEDENTES TEÓRICOS}

\section{Colaboración docente}

La colaboración docente es un factor esencial para promover e impulsar los procesos de mejora e innovación en los centros educativos (Sebastian y Allensworth, 2012). Lo anterior radica en que la colaboración posibilita nuevas oportunidades de desarrollo profesional fundadas en la reflexión compartida con otros colegas sobre los dilemas y problemáticas que suceden en su práctica (Butler y Schnellert, 2012). De este modo, el intercambio de recursos y la construcción de aprendizajes que se generan a partir de la colaboración constituyen una herramienta para la mejora de los procesos de enseñanza y aprendizaje (Little, 2002).

Con base a lo anterior, es posible definir la colaboración como un complejo entramado en el que confluyen y se relacionan en un contexto de aprendizaje interactivo un conjunto de valores, creencias, normas, modelos de relación, destrezas, patrones de acción, entre otros aspectos que el cuerpo docente comparte (González, 2014). Para que este entramado de procesos se pueda llevar a cabo y surjan nuevas herramientas pedagógicas que favorezcan el desarrollo profesional del personal docente, es necesario que el sistema escolar, en general, y la escuela, en particular, propicie entornos colaborativos donde predomine el apoyo mutuo, responsabilidades compartidas y la reflexión sistemática (Krichesky y Murillo, 2018), es decir, es necesario construir una cultura colaborativa.

Para propiciar una cultura colaborativa es fundamental generar condiciones estructurales mínimas tendientes a ayudar al cuerpo docente en el mejoramiento de sus prácticas, tal es el caso, por ejemplo, de establecer una distribución adecuada de tiempo lectivo/no lectivo (Organización para la Cooperación y el Desarrollo Económico, 2017). A nivel de la escuela, es clave el rol de los equipos directivos de los establecimientos educacionales (Szczesiul y Huizenga, 2014), ya que son ellos quienes deben promover el trabajo colaborativo entre los miembros del personal docente y no dificultarlo (Novoa, 2007). De este modo, 
una cultura que fomenta la colaboración requiere un equipo directivo que promueva políticas de formación que favorezcan el análisis, la reflexión y la mejora, tanto a nivel pedagógico como a nivel relacional. Asimismo, se requiere que el equipo directivo genere estructuras que permitan compartir objetivos, metodologías y, en especial, que garantice espacios de colaboración entre los miembros del cuerpo docente para fomentar el apoyo mutuo y la construcción de relaciones de trabajo significativas (Hugel, 2009).

Es importante destacar que una cultura colaborativa no solo favorece el crecimiento profesional del personal docente, sino que tiene un impacto en el aprendizaje del estudiantado, en especial de aquellos con discapacidad. Es así como el trabajo colaborativo facilita que el cuerpo docente pueda acoger y educar a toda la diversidad de estudiantes con que trabajan, diseñando actividades y utilizando metodologías para que todos y todas puedan aprender y participar. Es aquí donde el trabajo colaborativo con los y las profesionales de la educación especial es esencial, ya que esta práctica favorece la inclusión y permite que ambos profesionales aporten con su saber para avanzar y generar conocimientos y propuestas para construir aulas más inclusivas y diversas (Hugel, 2009).

\section{Diseño Universal para el Aprendizaje y Decreto 83}

El DUA consiste en un enfoque planteado por el Centro de Tecnología Especial Aplicada orientado a la superación de los contextos educativos discapacitantes que, a partir de los supuestos derivados del modelo médico, se caracterizan por un currículum homogéneo y rígido que fomenta procesos de estigmatización y exclusión de algunos grupos de estudiantes. Es así como el DUA se plantea como una perspectiva compuesta por principios fundamentados tanto en la investigación educativa como neurocientífica, junto con las posibilidades ofrecidas por las tecnologías digitales. Los tres principios básicos del DUA incluyen: a) proveer múltiples medios de compromiso, b) proveer múltiples medios de representación, y c) proveer múltiples medios de acción y expresión (Meyer y Rose, 2009; Meyer et al., 2014). Una de las principales ideas que sustentan estos principios tiene relación con comprender que el currículum, que desde este enfoque se integra de objetivos, métodos, materiales y evaluación (Hitchcock et al., 2002), debe caracterizarse por la flexibilidad para responder adecuadamente a la amplia diversidad del estudiantado y debe conducir al desarrollo de aprendices expertos. Los y las aprendices expertos son, en lo medular, personas que saben y quieren aprender (Center for Applied Special Technology, 2011).

Los principios del DUA anteriormente señalados permiten orientar el diseño, la selección y la aplicación de herramientas, métodos y entornos de aprendizaje (Meyer y Rose, 2009). Estos tres principios se encuentran fundamentados, considerando las aportaciones de la neurociencia, en tres redes neuronales de aprendizaje a nivel del sistema nervioso central: las redes afectivas, de reconocimiento y estratégicas. El estudiantado varía en múltiples aspectos, pero la variación en las dimensiones que reflejan los principios es especialmente crucial para el aprendizaje. En concreto, el primer principio (proveer múltiples medios de compromiso) está ligado a las redes afectivas y al porqué del aprendizaje, buscando con su aplicación que el alumnado se mantenga motivado y con propósito. El segundo principio (proveer múltiples medios de representación) se basa en las redes de reconocimiento y al qué del aprendizaje, además su consideración permite que los y las aprendices comprendan y sean capaces. Por su parte, el tercer principio (proveer múltiples medios de acción y expresión) se vincula con las redes estratégicas y al cómo del aprendizaje, esperando que el alumnado sea estratégico y orientado a metas.

Cada principio contiene tres pautas cuya finalidad consiste en crear y elegir de forma contextualizada y flexible herramientas, métodos y prácticas para la implementación del DUA (Meyer y Rose, 2009). Asimismo, se formula una pauta de acceso, construcción e internalización para cada principio (Center for Applied Special Technology, 2018). El primer principio contiene las pautas de provisión de opciones para: a) captar el interés, b) mantener el esfuerzo y la perseverancia y c) la autorregulación. Al segundo principio le fueron asociadas las pautas de provisión de opciones para: a) la percepción, b) el lenguaje y los símbolos 
y c) la comprensión. El tercer principio tiene asignadas las pautas de provisión para: a) la acción física, b) la expresión y la comunicación y c) las funciones ejecutivas. A su vez, es preciso puntualizar que cada pauta dispone de variados puntos de verificación de una naturaleza aún más concreta.

Los resultados de la revisión sistemática que Capp (2017) llevó a cabo sugieren que el DUA es una metodología de enseñanza efectiva para mejorar el aprendizaje para todo el estudiantado. Respecto al grupo de estudiantes con discapacidad, la instrucción basada en el DUA tiene el potencial de aumentar su participación y acceso al plan de estudios (Ok, Rao, Bryant y McDougall, 2017). Además, cabe señalar que las investigaciones informan la aplicación del DUA de diversas maneras y, por consiguiente, no existe un formato estándar para describir cómo se utiliza específicamente este marco (Rao, Ok, y Bryant, 2014). Los antecedentes acumulados en los últimos años muestran la importancia de planificar desde la perspectiva del DUA para que las clases puedan transformarse en una dirección inclusiva (Oliveira, Munster y Gonçalves, 2019).

En el contexto chileno, a nivel de política educativa, se ha considerado la aplicación gradual del DUA en las aulas en el nivel educativo parvulario y básico. El instrumento de tal normativa es el Decreto 83, el cual se concibe dentro del proceso más amplio de reforma educacional. Este Decreto tiene por propósito aprobar criterios y orientaciones de adecuación curricular y diversificación de la enseñanza (Ministerio de Educación, 2017). Este Decreto 83 se instala en el contexto de centros educativos que gestionan la diversidad a través de las ordenanzas del Decreto 170, el cual sigue vigente. Este decreto permite conformar en las escuelas los denominados Programas de Integración Escolar [PIE].

Los PIE son estrategias financiadas por el Estado que permiten que los establecimientos de educación regular puedan contratar a un equipo de profesionales, el que incluye típicamente educadores y educadoras diferenciales, fonoaudiólogos, fonoaudiólogas, psicólogas y psicólogos, para diagnosticar y apoyar a las y los estudiantes que presenten Necesidades Educativas Especiales. Desde el modelo médico, los PIE instalan el diagnóstico como única forma de acceder a financiamiento (Peña, 2013) y tienden a validar prácticas excluyentes (Mellado, Chaucono, Hueche y Aravena, 2017). En este contexto, los PIE son cuestionados por los propios docentes por el riesgo de psicopatologización (Núñez, Peña, González y Ascorra, 2020). Estos aspectos se vinculan a lógicas de enseñanza diferentes a las perseguidas por el DUA, haciendo coexistir influencias y demandas provenientes de miradas de la educación especial e inclusiva en el proceso de toma de decisiones del personal docente.

\section{MÉTODo}

El enfoque cualitativo guía los aspectos metodológicos de esta investigación, permitiendo comprender las perspectivas de las personas inscritas en contextos particulares (Flick, 2007). El foco principal de este estudio contempla acercarse a los conocimientos situados, es decir, aquellos abordados desde una posición determinada (Montenegro y Pujol, 2008) que, en este caso, refieren a las perspectivas y reflexiones que los educadores y las educadoras han desarrollado a partir de su participación experiencial en instancias colaborativas en el marco de la implementación del DUA en el aula.

\section{Participantes}

Se desarrolló una selección intencionada y por conveniencia, es decir, los y las participantes del estudio se escogieron con base en su fácil acceso y disponibilidad (Scribano, 2007). A su vez, se establecieron los siguientes criterios de selección para la muestra: a) contar con título profesional de Pedagogía en Enseñanza Básica; b) trabajar con al menos un curso de nivel parvulario o de primer o segundo ciclo básico/primario $\left(1^{\circ}\right.$ a $8^{\circ}$ año); c) que la escuela en donde desempeñara sus labores dispusiera de Programa de Integración 
Escolar [PIE] vigente, y d) que participara en reuniones periódicas de índole colaborativa donde se discutiera la implementación del DUA en su aula. De esta forma, se contó con la participación de 12 profesores y profesoras que ejercían en la Región Metropolitana de Chile que se desempeñaban en escuelas municipales. Cabe puntualizar que nueve participantes fueron mujeres y tres hombres.

\section{Procedimientos de producción de información}

Se aplicó una entrevista cualitativa a cada participante en función de un guion flexible que consistía fundamentalmente en dos etapas. La primera etapa se diseñó bajo los principios de la entrevista episódica como técnica de producción de datos cualitativos. En efecto, se formularon preguntas que tenían por finalidad invitar a presentar narraciones de las situaciones de interés (Flick, 2007) que, en este estudio, corresponden a las experiencias de colaboración asociadas a la aplicación del DUA. El componente narrativo en las entrevistas realizadas se justifica al considerar que los relatos otorgan información contextual e intencional de manera integrada. Al respecto, Bruner $(1990 ; 2004)$ refiere que las narraciones se configuran en un paisaje dual que, por un lado, incluyen las intenciones y pensamientos de la mente y, por otro, incorporan acciones y datos de contexto. Además, las experiencias de colaboración constituyen una práctica social y, por dicha razón, es la comprensión del contexto sociohistórico el que permite dar significado a las acciones y sucesos que allí ocurren (Wenger, 2002).

La segunda etapa de la entrevista cualitativa se caracterizó por el empleo de preguntas que rescataran la modalidad paradigmática del pensamiento de los participantes en relación con el tema de investigación. De acuerdo con Bruner (2004), esta modalidad alude a los significados que tienen por función desarrollar descripciones y explicaciones generales. Este concepto se relaciona con el constructo de teoría subjetiva propuesto por Groeben (1988), el cual es comprendido como una unidad compleja estructurada argumentativamente para alcanzar funciones descriptivas, explicativas, predictivas y aplicativas. En el contexto de esta investigación, a los profesores y las profesoras participantes se les solicitó brindar sus significados paradigmáticos en término de descripciones, razones, opiniones, explicaciones y argumentos en torno a las experiencias de colaboración en la aplicación del DUA.

El acceso tanto de significados narrativos como paradigmáticos es considerado una manera de responder a la complejidad intrínseca que conforma las reflexiones como objeto de estudio. De este modo, el carácter situado de las reflexiones (Putnam y Borko, 2000) se abordó en la producción discursiva de experiencias que se plasman en una modalidad narrativa (Flick, 2007; Ricoeur, 1995). Por otro lado, el cuerpo de herramientas argumentativas, conceptuales e interpretativas que se aplican y fundamentan a dichas experiencias y prácticas (Dewey, 1933; Schön, 1986), fue trabajado mediante la promoción de ideas paradigmáticas.

\section{Procedimiento de análisis de información}

Las transcripciones de datos narrativos y paradigmáticos provenientes de las entrevistas realizadas fueron analizadas en función de los lineamientos de la Teoría Fundamentada de Strauss y Corbin (2002). Para alcanzar resultados de mayor consistencia, se recurrió a la triangulación de investigadores, procedimiento que involucró una activa participación de los miembros del equipo de investigación a lo largo del proceso de análisis de los datos a través de reuniones periódicas. En una primera aproximación, se analizó mediante microanálisis (codificación abierta y axial) el material de acuerdo con su carácter narrativo y paradigmático, estableciéndose categorías y relaciones parciales. Posteriormente, se empleó la codificación selectiva para elaborar un esquema que integró de manera articulada las producciones analíticas de las dos modalidades de pensamiento revisadas y que, en conjunto, permitiera dar cuenta de la reflexión de las profesoras y los profesores. 
Las operaciones analíticas señaladas fueron llevadas a cabo en el programa computacional Atlas.ti, ya que permite facilitar el proceso analítico, especialmente al utilizar la Teoría Fundamentada (San Martín, 2014). Así, este programa es útil para avanzar en labores de abstracción y conceptualización a partir de procesos inductivos y exploratorios aplicados a datos textuales (Penalva, Alaminos, Francés y Santacreu, 2015), sobre la base de la acción interpretativa llevada a cabo por los investigadores (Padilla y Vega, 2014). En el contexto de este estudio, este programa permitió desarrollar efectivamente una codificación inicial, una categorización consolidada y establecer familias de categorías que, como se verá más adelante, fueron designadas como niveles. El despliegue visual de las categorías facilitó la interpretación conjunta del equipo investigador sobre sus relaciones, al igual que la conformación del esquema final que sintetiza los fenómenos estudiados.

\section{Resultados}

A partir del análisis de las reflexiones de los profesores y las profesoras respecto de sus experiencias de colaboración profesional para introducir el DUA en las prácticas educativas, se delimitaron variadas categorías que fueron situadas en tres niveles. Estos niveles identificados son: nivel institucional, de relación profesional y de aula. Los niveles se encuentran representados en la Figura 1, en la cual se muestra la distribución de las categorías construidas tras la codificación analítica y, junto con lo anterior, se expresan sus principales relaciones. Es necesario mencionar que la categoría eje desde la cual pueden comprenderse las demás se trata de la actividad colaborativa profesional. Tal categoría está condicionada y tensionada fuertemente por las condiciones institucionales en donde se sitúa. Producto de esta situación, la descripción de los resultados comenzará con el nivel institucional, continuará con el nivel de relación profesional y concluirá con el nivel de aula. 


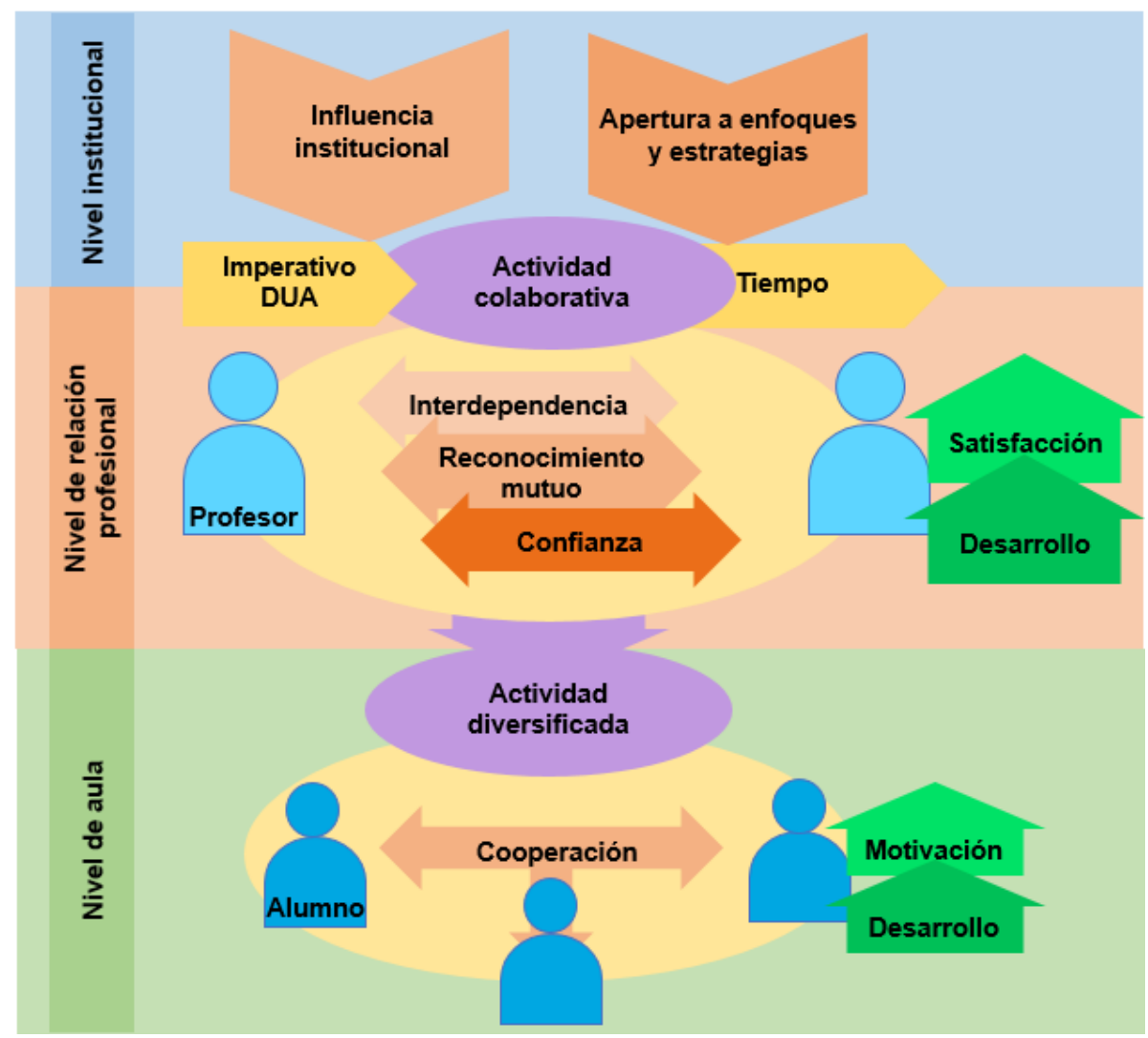

FIGURA 1.

Diagrama de las reflexiones de las profesoras y los profesores sobre sus experiencias de colaboración en el marco del Diseño Universal para el Aprendizaje

Fuente: Elaboración propia.

\section{Nivel institucional}

Los y las participantes afirman que la interacción colaborativa entre agentes educativos es facilitada o impedida por este nivel, en el cual reconocen, a su vez, distintos subniveles. En la categoría influencia institucional se posiciona el subnivel de centro educativo, donde el clima social y los valores institucionales juegan un rol central. Es así como un clima social positivo que entrega valor a la colaboración es percibido como un impulsor de instancias colaborativas productivas. En la construcción de este clima que promueve colaboración, se entiende que una adecuada gestión de las autoridades del establecimiento escolar sería una condición indispensable. Junto con lo anterior, el establecimiento de criterios compartidos por el personal del centro también permitiría desarrollar un terreno de confianza para que el cuerpo docente mantuviera encuentros significativos.

En esta misma categoría, se reconoce otro subnivel de naturaleza más general que el anterior. Este subnivel sería, de acuerdo con la representación del personal docente, el sistema estatal. Las reflexiones de los miembros del grupo participante son unívocas al cuestionar la labor del Estado para equilibrar, por un lado, la demanda de diversificación de la enseñanza y, por otro, la disponibilidad de recursos de apoyo y adecuadas condiciones laborales. Básicamente, el recurso más solicitado para encarar esta demanda de manera satisfactoria consiste en disponer formalmente de más espacios de tiempo. Por otro lado, se presentan también críticas respecto al diseño de los programas de estudio y las pruebas estandarizadas, dado que se percibe que estos no entregan el suficiente margen para la diversificación de la enseñanza. 
(El DUA), a veces, se contrapone a los programas de estudios, porque yo te hablo desde el área de lenguaje. Porque el programa de estudio te exige que puedas trabajar los cuatro ejes que se trabajan en lenguaje. Pero, resulta que, si yo le estoy pidiendo a un niño desde lo que el elija hacer, que me haga un cuento y me va a dibujar, no me está escribiendo...pero lo que el programa dice es que tiene que tener una redacción, una ortografía (Profesora 7, comunicación personal, 17 de mayo, 2019).

Por su parte, algunos participantes entienden el DUA como un marco general, el cual puede nutrirse con otros enfoques pedagógicos, concepciones sobre los procesos de enseñanza y aprendizaje, y estrategias educativas. En la Figura 1 esta interpretación está representada en la categoría Apertura a enfoques y estrategias. Esta apertura se traduce en la búsqueda expresa de nuevos enfoques que permitan nutrir las reflexiones en colaboración y, de esta manera, producir ideas pedagógicas con matices innovadores y flexibles. Esta orientación conlleva a valorar tanto los referentes teóricos generados por la comunidad científica como los espacios formativos que permiten apropiarse de estos referentes y darles utilidad. Las estrategias pedagógicas asociadas al aprendizaje por proyectos son especialmente estimadas como coherentes con los principios del DUA.

Yo veo que la única forma de trabajar (el DUA) es trabajar proyectos. Porque en los proyectos los niños deben investigar, tiene que haber una necesidad, tienen que formular hipótesis, tienen que hacer múltiples cosas, son miles de habilidades y estrategias. Entonces, de la única forma que yo lo veo para que lo puedan implementar (DUA), es trabajando proyectos (Profesora 3, comunicación personal, 9 de abril, 2019).

Adicionalmente, el grupo participante visualiza que la conformidad con métodos de enseñanza tradicionales opera como un obstaculizador a los esfuerzos de diversificación y colaboración entre colegas. Este elemento se entiende como una resistencia difícil de sobrellevar, ya que al apego irrestricto a los métodos convencionales le subyace una larga trayectoria de aprendizaje profesional en su utilización.

\section{Nivel de relación profesional}

Los elementos de este nivel se encuentran condicionados por el imperativo normativo de diversificar la enseñanza a través de los principios del DUA. Aunque tal directriz es abordada con mayor o menor familiaridad por parte del grupo participante, este asume con unanimidad que es necesario intensificar procesos de reflexión conjunta en el contexto. La actividad colaborativa que supone la reflexión conjunta se concreta en varios procesos típicos del trabajo docente, tales como: codocencia, planificación, elaboración de materiales y evaluaciones y, además, detección de necesidades del estudiantado. Los participantes subrayan la necesidad de contar con tiempos lo suficientemente significativos para desarrollar dichos procesos. Dado que los espacios formales son percibidos como escasos, varios profesores y profesoras comentan que emplean tiempos alternativos, tales como los recreos o, inclusive, momentos fuera de la jornada laboral.

Los miembros del grupo participante identifican múltiples aspectos de la relación entre colegas que, al estar presentes, permiten una colaboración connotada como favorable. Un primer aspecto es la interdependencia profesional, la cual depende, en este contexto, de la adscripción de la intención del DUA por superar estrategias de enseñanza de talla única y estandarizadas para, de esta manera, asegurar la participación y aprendizaje de todo el alumnado. Este elemento implica, además, que se requiere un trabajo basado en la responsabilidad compartida hacia este objetivo y una adherencia a un proceso conjunto de toma de decisiones; cada quien aportando desde sus saberes y competencias.

El reconocimiento mutuo, como segundo aspecto de una colaboración favorable, está estrechamente relacionado con el anterior. Este aspecto se cimienta en el supuesto compartido por los participantes de que una mayor diversificación de la enseñanza se logrará mediante múltiples aportaciones. Se trata, por tanto, de valorar las fortalezas y conocimientos de los demás para construir una práctica educativa de mayor riqueza y coherencia con el DUA. En este proceso, la confianza es fundamental y, por este motivo, es identificada como un tercer elemento de importancia. Según la perspectiva de los docentes, la confianza permitiría reconocer 
dificultades, errores, carencias y la necesidad de complementar las propias ideas y labores. Sin la confianza, las actividades de colaboración serían mínimamente aprovechadas.

Hay mucha confianza en ese aspecto, de pedir ayuda, de comentar los errores. Salimos de la sala no como con frustración, ni con rabia, como salíamos antes, sino que "Chuta, no me resultó esto ¿En qué fallé?" Con esa sensación. Así lo comentamos. "Sabes hice esto y no me resultó ¿qué creí tú que podríamos hacer?” Estoy super contenta este año (Profesora 11, comunicación personal, 6 de junio, 2019).

Como puede constatarse con la lectura del extracto anterior, las y los profesores han experimentado satisfacción mediante las dinámicas reforzadas de colaboración tras comprometerse con los principios planteados por el DUA. Tal sentimiento de satisfacción se produce, de acuerdo con la mirada de los docentes, por los resultados en los aprendizajes y motivación de las y los estudiantes y, especialmente, por los vínculos de colaboración que son construidos y fortalecidos entre colegas. Adicionalmente, los maestros perciben que su participación continua en espacios de colaboración orientados a fomentar la diversificación de sus prácticas les ha permitido desarrollar múltiples habilidades y conocimientos. Por tanto, los docentes conciben estos espacios como contextos de aprendizaje y formación profesional.

Se ha ido como contagiando, es como un virus. A partir de los éxitos que se van teniendo. Los que estamos trabajando así salimos contentos de la sala de clases. Yo no veo ninguna colega que trabaje esto que llegue enojada, porque no trabajas en función del éxito sino en relación a la colaboración y colaboramos todos. Aprender a través de la colaboración y lo propone el DUA (Profesora 2, comunicación personal, 9 de abril, 2019).

\section{Nivel de aula}

El producto central del trabajo profesional colaborativo es, desde la visión de las y los profesores, una planificación didáctica con mayor diversificación en comparación con un proceso de diseño solitario. Muchos de los profesores y las profesoras que trabajan regularmente de este modo han reconocido el valor del aprendizaje cooperativo para maximizar la diversificación de estrategias, recursos y opciones para las y los estudiantes. Además, la apertura a transitar a una implementación habitual del aprendizaje cooperativo también está mediada, de acuerdo con algunos de los docentes, por la experimentación de una colaboración profesional satisfactoria. Producto de adherir a los principios del DUA, muchos maestros tienden a utilizar esta estrategia de aprendizaje de manera muy frecuente, especialmente trabajando con grupos heterogéneos:

Todo se hace en grupo y todos los grupos son heterogéneos, hombres, mujeres. No tenemos separados a los niños, aquí están los PIE, aquí están los que son más aventajados, no está ese criterio (Profesora 11, comunicación personal, 6 de junio, 2019).

Las consecuencias que perciben los maestros en torno a la introducción de más actividades diversificadas pueden agruparse fundamentalmente en dos tipos: desarrollo y motivación. Respecto al primero, las y los profesores aseveran que las actividades diversificadas realizadas de manera cooperativa tienen un potente impacto en el desarrollo integral del estudiantado, es decir, en los resultados académicos y, particularmente, en aspectos valóricos y actitudinales. También, la autonomía de los y las estudiantes es mayor, al igual que su participación. En segundo término, el grupo entrevistado señala que la motivación del alumnado se acrecienta si es comparada con formatos de enseñanza de índole tradicional, transmisivo y de talla única. Las razones identificadas por las entrevistas y los entrevistados que permiten explicar esta tendencia corresponden a la posibilidad de elección de los y las estudiantes entre variadas alternativas basadas en sus intereses y las estructuras cooperativas de participación. Además, las y los docentes plantean que se logra involucrar en múltiples actividades a estudiantes que con una estructura uniforme de enseñanza quedaban típicamente excluidos. 
Es distinto porque es más lúdico. Ellos quieren participar, participan más. Por ejemplo, los niños que son más tímidos se atreven más. Se atreven más que en una clase expositiva... Les rompe un poco el esquema y ellos esperan la clase (Profesora 6, comunicación personal, 16 de mayo, 2019).

En el contexto del aula, los participantes mencionan variadas acciones que les han servido para avanzar en una implementación progresiva del DUA. En primer lugar, algunos profesores y profesoras se refieren a la utilidad de inscribir tareas escolares específicas y típicas de sus disciplinas en actividades grupales de naturaleza más compleja, como obras teatrales, proyectos o actos. En tales espacios, se considera necesario presentar diferentes roles que puedan asumir los y las estudiantes de acuerdo con sus intereses. Asimismo, varios participantes indican que es posible brindar múltiples artefactos de apoyo que quedan, en buena parte, a elección del grupo de estudiantes en función de las metas educativas. También, se señala la conveniencia de dar la oportunidad a los propios estudiantes de construir estos artefactos, agregando elementos asociados a las manualidades en diversas disciplinas. Por supuesto, la apertura a la incorporación y uso de distintas tecnologías es referido por los participantes como un aspecto ineludible, al igual que rescatar las opiniones de las niñas y niños respecto a cómo plasmar concretamente las actividades de aprendizaje.

Estos y otros elementos puntualizados tienen el potencial de acrecentar significativamente, desde la mirada del profesorado entrevistado, la motivación de las y los estudiantes. Según algunos participantes, la capacidad de tales medidas viene dada al permitir suplir necesidades personales de distinta naturaleza en el alumnado, como la de sentirse valorado y apoyado, obtener reconocimiento social, cubrir intereses variados, asumir responsabilidades, lograr metas, construir cosas de propia autoría, entre otras.

\section{Conclusiones}

Esta investigación ha mostrado diversas reflexiones del cuerpo docente, que ha experimentado dinámicas colaborativas conducentes a diversificar sus prácticas educativas. Estas reflexiones abarcan elementos y procesos que se ubican en tres niveles diferentes y que gravitan en la actividad colaborativa entre los profesores y las profesoras. Los resultados desprendidos de las reflexiones analizadas dan cuenta que tal actividad colaborativa está condicionada por factores contextuales institucionales, como las políticas educativas, las dinámicas del centro educativo y las aportaciones de las ciencias de la educación. La naturaleza de esta actividad colaborativa, cuando es satisfactoria, se caracteriza tanto por la interdependencia de perspectivas y saberes como por la confianza en las aportaciones de los demás para lidiar con el desafío de la diversificación. Precisamente, la diversificación de la enseñanza en el aula se plasma con mayor coherencia en lógicas cooperativas, las que conducen a niveles más altos de motivación y desarrollo en el alumnado y que tienen como condición la colaboración profesional del magisterio.

Si bien cada uno de los aspectos que aparecen en las reflexiones del profesorado son fenómenos de notable complejidad por sí mismos, los resultados expuestos muestran una estructura general y articulada del carácter de la reflexión de aquellas profesoras y profesores con experiencias favorables en el intento de diversificar la enseñanza a través de la colaboración profesional. De esta manera, esta investigación permite interrelacionar los diversos elementos que se desarrollan en el pensamiento reflexivo del profesorado respecto a la temática tratada en un todo integrado. Por consiguiente, los elementos identificados en este estudio que componen la reflexión del cuerpo docente promueven o limitan a los demás, posicionándose cada aspecto como un constituyente indispensable. Así, un elemento desvirtuado puede, por sí solo, degradar la experiencia satisfactoria aludida por los y las docentes, ya sea que tome forma como una influencia institucional restrictiva, un rechazo a enfoques pedagógicos innovadores, un aislamiento profesional, una fuerte actitud de desconfianza, un desconocimiento de la aportación del otro o una aplicación invariable de actividades pedagógicas individualistas.

Acerca del nivel institucional, este implica posicionar los resultados en el contexto del sistema escolar chileno, a partir de lo cual se derivan dos conclusiones. Primeramente, a nivel de la institución escolar 
los resultados muestran la relevancia del liderazgo directivo en la construcción de culturas colaborativas basadas en relaciones de confianza. Por tanto, el esfuerzo sostenido en la instalación y facilitación de culturas profesionales colaborativas en los centros educativos se posiciona como una condición sine qua non para concretar prácticas de diversificación de la enseñanza. Un ejemplo de la utilidad de un soporte cultural y administrativo a favor de la colaboración profesional se relaciona con la posibilidad de propiciar espacios formativos, en los cuales se discutan aportes del ámbito científico y enfoques pedagógicos innovadores para enriquecer las prácticas educativas inclusivas.

En segundo lugar, esta investigación, como otras anteriores (Núñez, Peña, González y Ascorra, 2020), sugiere que el PIE, política mediante la cual se incorpora el DUA en Chile, constituye una instancia formal que tiende a facilitar la colaboración entre los y las profesionales de la educación. Sin embargo, los resultados también plantean que las dinámicas colaborativas entre el cuerpo docente son tensionadas por prescripciones de la política educativa. Puntualmente, los escasos tiempos no lectivos y la rigidez de un currículum nacional evaluado a través de una prueba censal con altas consecuencias ${ }^{[1]}$, son representados desde el profesorado como serios obstáculos para una colaboración efectiva. En este sentido, tomando en cuenta la importancia de los procesos colaborativos en el contexto escolar, parece urgente dotar de mayor coherencia sistémica el conjunto de políticas que regulan el trabajo del profesorado.

Respecto al nivel de relación profesional, es posible establecer dos conclusiones principales. La primera refiere a la importancia que posee para la diversificación de la enseñanza la reflexión entre los miembros del cuerpo docente, la cual se convierte en una estrategia clave para trabajar con la diversidad en el aula. En este sentido, cabe señalar que la práctica de los y las docentes se caracteriza por ser incierta, cambiante, singular, compleja, presentando conflictos en la definición de metas y la elección de medios para lograrlas (Schön, 1983). Por lo tanto, para abordar la complejidad de la práctica educativa y lograr que todas y todos los estudiantes aprendan, es necesario que el profesorado actúe como expertos adaptativos, ya que son las necesidades cambiantes del estudiantado, los objetivos particulares que se buscan alcanzar en un determinado momento y los eventos inesperados del aula lo que marca su labor (Hammerness et al., 2005). De esta manera, la reflexión conjunta permite construir alternativas pedagógicas que respondan a la diversidad del alumnado, adaptarlas y evaluarlas en contexto, tareas de suma relevancia en el desarrollo del DUA en el aula.

Por su parte, la segunda conclusión desprendida a partir de los resultados obtenidos apunta a cómo la colaboración permite al cuerpo docente mejorar sus habilidades, convirtiéndose en un dispositivo que promueve el aprendizaje y la formación profesional. De acuerdo con los principales resultados en este nivel de análisis, este proceso formativo de apropiación de herramientas y co-construcción de recursos para la diversificación de la práctica educativa depende de que los participantes de las instancias reflexivas conciban la relación desde la interdependencia, la confianza y el reconocimiento mutuo. En otras palabras, las potenciales contribuciones de los pares al quehacer educativo se cimientan en la valoración del otro y en comprender que una adecuada praxis profesional en el ámbito educativo asociado al DUA rechaza el aislacionismo.

A nivel de aula, es posible llegar a tres conclusiones a partir de los resultados obtenidos. En primer lugar, la planificación se reconoce como la actividad colaborativa por parte de los agentes educativos que vertebra la diversificación de la enseñanza. Por supuesto, esta idea es coherente con la premisa del DUA de diseñar con antelación actividades de aprendizaje para atender la diversidad en el aula (Meyer et al., 2014). De esta manera, la capacidad de maximizar la diversificación de enseñanza descansa fundamentalmente en potenciar el encuentro de distintas perspectivas e ideas por parte de las y los profesionales involucrados.

En segundo lugar, la colaboración satisfactoria en la construcción e implementación de actividades basadas en el DUA se relaciona con la afinidad o compromiso con otros elementos que describen un aula inclusiva. En particular, se vislumbra a partir de los resultados que la implementación del DUA se vincula y es potenciada con organizaciones cooperativas, recolección de los intereses y opiniones del estudiantado, uso de diversas capacidades que estimulen el desarrollo integral, entre otros aspectos. Además, muchas y muchos docentes encuentran en las ideas del aprendizaje basado en proyectos, en el cual se emplean actividades auténticas que 
incluyen los intereses y la elección del alumnado (Barron y Darling-Hammond, 2016), formas de concreción de los principios del DUA. Este aspecto demuestra que el DUA no es una estrategia pedagógica concreta que solvente, por sí misma, el conjunto de necesidades de la toma de decisiones del cuerpo docente, sino que se concibe como un razonamiento de trabajo educativo con la diversidad que requiere, en su materialización, el apoyo de variadas perspectivas que pueden ofrecer las ciencias de la educación.

\section{Recomendaciones}

El cuerpo de hallazgos de la presente investigación permite formular distintas recomendaciones, las cuales pueden orientar procesos de mejora escolar para introducir colaborativamente el DUA. Una primera sugerencia apunta a comprender la implementación del DUA como un encuentro de miradas entre agentes educativos, el cual avanza a partir de procesos reflexivos e instancias formativas donde se generan adaptativamente recursos, especialmente en espacios de planificación de actividades escolares. La segunda implicación a nivel educativo viene dada por la necesidad de crear las condiciones para que estos encuentros reflexivos tengan lugar y sean efectivos. Las condiciones de tiempo y espacio, la coherencia de la política educativa, una cultura que apoye la colaboración, la interdependencia, la confianza y, además, los espacios de discusión sobre enfoques pedagógicos innovadores son, en esencia, los aspectos más relevantes según los y las educadoras. Adicionalmente, las perspectivas de los participantes de este estudio también muestran la conveniencia de entender el DUA como un marco referencial que puede ser implementado de mejor manera en combinación con enfoques de aprendizaje cooperativos y basados en proyectos.

Por último, se reconocen fundamentalmente dos limitaciones de este estudio que pueden configurar proyecciones investigativas de importancia. Primero, como los participantes de este estudio fueron solo profesores y profesoras de Enseñanza Básica, sería propicio incorporar paralelamente las visiones de otros agentes y actores educativos. Por ejemplo, sería un importante aporte recoger conjuntamente, en el contexto de una misma investigación, las reflexiones de los profesores y las profesoras de educación especial, las y los asistentes de aula, los apoderados y las apoderadas, estudiantado, psicólogas y psicólogos educacionales, entre otros. En segundo lugar, convendría analizar también las experiencias y reflexiones de profesores y profesoras que rechazan tanto los procesos colaborativos de carácter profesional como la implementación del DUA en sus aulas. Comprender los procesos argumentativos que configurarían sus reflexiones podría ofrecer información valiosa para guiar procesos de mejora escolar teniendo presente las inevitables resistencias de cualquier cambio educativo.

\section{Agradecimientos}

Financiamiento asociado: Agencia Nacional de Investigación y Desarrollo / Programa de Becas / Beca de Doctorado en el Extranjero BECAS CHILE 72200341 y 72210436 / Beca de Doctorado Nacional 21212101

\section{ReFERENCIAS BibliográFicas}

Ainscow, M. (1995). Necesidades especiales en el aula. Guia para la formación del profesorado. Madrid: Narcea.

Al-Azawei, A., Serenelli, F. y Lundqvist, K. (2016). Universal Design for Learning (UDL): A Content Analysis of Peer-Reviewed Journal Papers from 2012 to 2015. Journal of the Scholarship of Teaching and Learning, 16(3), 39-56. doi: https://doi.org/10.14434/josotl.v16i3.19295

Barron, B., y Darling-Hammond, L. (2016). Perspectivas y desafíos de los enfoques del aprendizaje basados en la indagación. En OECD, La naturaleza del aprendizaje. Usando la investigación para inspirar la práctica (pp. 158-183). Panamá: OECD/Unesco/Unicef. 
Blanco, R. (2006). La equidad y la inclusión social: uno de los desafíos de la educación y la escuela hoy. Revista Electrónica Iberoamericana sobre Calidad, Eficacia y Cambio en Educación, 4(3), 1-15. Recuperado de: https:// repositorio.uam.es/handle/10486/660828

Bruner, J. (1990). Actos de Significado. Más allá de la revolución cognitiva. Madrid: Alianza.

Bruner, J. (2004). Realidad mental y mundos posibles. Los actos de imaginación que dan sentido a la experiencia. Barcelona: Gedisa.

Butler, D. L., y Schnellert, L. (2012). Collaborative inquiry in teacher professional development. Teaching and Teacher Education, 28(8), 1206-1220. doi: https://doi.org/10.1016/j.tate.2012.07.009

Cabezas, V., Medeiros, M., Inostroza, D. Gómez, C. y Loyola, V. (2017). Organización del Tiempo Docente y su Relación con la Satisfacción Laboral: Evidencia para el Caso Chileno. Education Policy Analysis Archives/Archivos Analiticos de Politicas Educativas, 25(64), 1-33. doi: http://dx.doi.org/10.14507/epaa.25.2451

Capp, M. (2017). The effectiveness of universal design for learning: a meta-analysis of literature between 2013 and 2016. International Journal of Inclusive Education, 21(8), 791-807. doi: https://doi.org/10.1080/13603116.20 17.1325074

Center for Applied Special Technology. (2011). Universal Design for Learning guidelines version 2.0. Wakefield, MA: CAST.

Center for Applied Special Technology. (2018). Universal Design for Learning Guidelines version 2.2. Wakefield, MA: CAST. Recuperado de http://udlguidelines.cast.org

Dewey, J. (1933). How we think. London: D.C. Health Co.

Echeita, G. (2006). Educación para la inclusión o educación sin exclusiones. Madrid: Narcea.

Flick, U. (2007). Introducción a la investigación cualitativa. Madrid: Morata.

Florian, L. (2017). Teacher Education for the Changing Demographics of Schooling: Inclusive Education for Each and Every Learner. En L. Florian y N. Pantić (Eds.), Teacher education for the changing demographics of schooling. Issues for Research and Practice (pp. 9-20). Switzerland: Springer International Publishing AG.

González, E. (2014). Una mirada del trabajo colaborativo en la escuela primaria desde las representaciones sociales. $R a$ Ximhai, 10(5), 115-134. doi: https://doi.org/10.35197/rx.10.03.e1.2014.08.jg

Gravel, C., Le Bossé, Y., y Fournier, G. (2019). L'écart entre la valorisation de la collaboration entre enseignant.e-s et la difficulté de sa mise en oeuvre formelle. Revue Des Sciences de L'éducation, 45(1), 215-240. doi: https://doi. org/10.7202/1064612ar

Groeben, N. (1988). Explikation des Konstrukts "Subjektive Theorie”. En N. Groeben, D. Wahl, J. Schlee, y B. Scheele (Eds.), Das Forschungsprogramm Subjektive Theorien: eine Einführung in die Psychologie des reflexiven Subjekts (pp. 17-23). Germany: Francke Verlag.

Hammerness, K., Darling-Hammond, L., Bransford, J., Berliner, D., Cochran-Smith, M., McDonald, M. y Zeichner, K. (2005). How teachers learn and develop. En L. Darling-Hammond y J. Bransford (Eds), Preparing teachers for a changing world (pp. 358-389). Estado Unidos: Jossey-Bass.

Hauge, K., y Wan, P. (2019). Teachers' collective professional development in school: A review study. Cogent Education, 6(1), 1-22. doi: https://doi.org/10.1080/2331186X.2018.1522781

Hitchcock, C., Meyer, A., Rose, D., y Jackson, R. (2002). Providing new access to the general curriculum. universal design for learning. Teaching Exceptional Children, 35(2), 8-17. doi: https://doi.org/10.1177/004005990203 500201

Hugel, T. (2009). El trabajo colaborativo entre el profesorado como estrategia para la inclusión. En C. Giné (Coord.), La educación inclusiva: de la exclusión a la plena participación de todo el alumnado (pp. 81-94). España: Horsori.

Knox, J., y Anfara, V. (2013). What research says: Understanding job satisfaction and its relationship to student academic performance. Middle School Journal, 44(3), 58-64. Recuperado de https://www.jstor.org/stable/41 763131

Krichesky, G., y Murillo, F (2018). La colaboración docente como factor de aprendizaje y promotor de mejora. Un estudio de casos. Educación XX1, 21(1), 135-155. doi: https://doi.org/10.5944/educxx1.20181 
Karina del Carmen Moreno Díaz, et al. Procesos argumentativos que conforman las reflexiones del Pr...

Little, J. (2002). Locating learning in teachers' communities of practice: opening up problems of analysis in records of everyday work. Teaching and Teacher Education, 18(8), 917- 946. doi: https://doi.org/10.1016/S0742-051 $\mathrm{X}(02) 00052-5$

Mellado, M. E., Chaucono, J. C., Hueche, M. y Aravena, O. (2017). Percepciones sobre la educación inclusiva del profesorado de una escuela con Programa de Integración Escolar. Revista Educación, 41(1), 119-132. doi: http ://dx.doi.org/10.15517/revedu.v41i1.21597

Meyer, A. y Rose, D. (2009). A policy reader in universal design for learning. Boston, MA: Harvard Education Press.

Meyer, A., Rose, D., y Gordon, D. (2014). Universal design for learning: Theory and practice. Wakefield MA: Center for Applied Special Technology.

Ministerio de Educación. (2017). Orientaciones sobre estrategias diversificadas de enseñanza para educación básica, en el marco del Decreto 83/2015. Santiago: Ministerio de Educación.

Montenegro, M., y Pujol, J. (2008). Derivas y actuaciones: aproximaciones metodológicas. En A. Gordo y A. Serrano (Coomps.), Estrategias y prácticas cualitativas de investigación social (pp. 75-94). Madrid: Pearson.

Novoa, A. (2007). El profesor hoy. La participación, la coparticipación y la prudencia. Cuadernos de Pedagogía, 374 , 21-25. Recuperado de: https://bit.ly/3brJTpa

Núñez, G., Peña, M., González, B., y Ascorra, P. (2020). Una mirada desde la inclusión al Programa de Integración Escolar (PIE) en escuelas rurales chilenas: un análisis de casos. Revista Colombiana de Educación, 1(79), 371-392. doi: https://doi.org/10.17227/rce.num79-9725

Ok, M., Rao, K., Bryant, B., y McDougall, D. (2017). Universal design for learning in pre-k to grade 12 classrooms: A systematic review of research. Exceptionality, 25(2), 116-138. doi: https://doi.org/10.1080/09362835.2016 .1196450

Oliveira, A., Munster, M. y Gonçalves, A. (2019). Universal Design for Learning and Inclusive Education: a Systematic Review in the International Literature. Revista Brasileira de Educação Especial, 24(4), 627-640. doi: http://dx. doi.org/10.1590/s1413-65382519000400009

Onrubia, J. (1993). La atención a la diversidad en la enseñanza secundaria obligatoria: Algunas reflexiones y criterios psicopedagógicos. Aula de innovación educativa, 12, 45-49. Recuperado de http://www.altascapacidadescse.org /pdf/atenc_diversidad_secundari.pdf

Organización para la Cooperación y el Desarrollo Económico. (2017). Evaluaciones de Políticas Nacionales de Educación. Educación en Chile. Santiago: OCDE, Fundación SM.

Oyarzún, C., Soto, R. y Moreno, K. (2019). Tensiones del trabajo docente en un contexto gerencialista: una revisión de literatura sobre el caso chileno. Educação (UFSM), 44. 1-27. doi: http://dx.doi.org/10.5902/1984644434888

Padilla, J. y Vega, P. (2014). Teoría fundamentada y sus implicaciones en investigación educativa: el caso de Atlas.ti. Revistas de investigaciones UNAD, 13(1), 23-39. doi: https://doi.org/10.22490/25391887.1129

Penalva, C., Alaminos, A., Francés, F. y Santacreu, O. (2015). La investigación cualitativa. Técnicas de investigación y análisis con Atlas.ti. Ecuador: Pydlos.

Peña, M. (2013). Análisis crítico de discurso del Decreto 170 de Subvención Diferenciada para Necesidades Educativas Especiales: El diagnóstico como herramienta de gestión. Psicoperspectivas, 12(2), 93-103. doi: https://doi.org/: 10.5027/psicoperspectivas-vol12-issue2-fulltext-252

Putnam, R. y Borko, H. (2000). What do new views of knowledge and thinking have to say about research on teacher learning? Educational Researcher, 29(1), 4-15. doi: https://doi.org/10.3102/0013189X029001004

Rao, K., Ok, M., y Bryant, B. (2014). A review of research on universal design educational models. Remedial and special education, 35(3), 153-166. doi: https://doi.org/10.1177/0741932513518980

Ricoeur, P. (1995). Tiempo y narración I: Configuración del tiempo en el relato histórico. México: Siglo XXI.

San Martín, D. (2014). Teoría fundamentada y Atlas.ti: recursos metodológicos para la investigación educativa. Revista Electrónica de Investigación Cualitativa, 16(1). Recuperado de https://redie.uabc.mx/redie/article/view/727/8 91 
Sandoval, M. (2008). La colaboración y la formación del profesorado como factores fundamentales para promover una educación sin exclusiones. Contextos Educativos, 11, 149-159. doi: https://doi.org/10.18172/con.600

Scribano, A. (2007). El proceso de investigación social cualitativo. Buenos Aires: Prometeo Libros.

Schön, D. (1983). El profesional reflexivo: cómo piensan los profesionales cuando actúan. Barcelona: Paidos.

Schön, D. (1986). La formación de profesionales reflexivos. Hacia un nuevo diseño de la enseñanza y el aprendizaje en las profesiones. Barcelona: Paidos

Sebastian, J. y Allensworth, E. (2012). The influence of principal leadership on classroom instruction and student learning: A study of mediated pathways to learning. Educational Administration Quarterly, 48(4), 626-663. doi: https://doi.org/10.1177/0013161X11436273

Strauss, A. y Corbin, J. (2002). Bases de la Investigación Cualitativa. Medellín: Editorial de la Universidad de Antioquia.

Szczesiul, S., y Huizenga, J. (2014). The burden of leadership: Exploring the principal's role in teacher collaboration. Improving Schools, 17(2), 176-191. doi: https://doi.org/10.1177/1365480214534545

Villegas, A., Ciotoli, F. y Lucas, T. (2017). A framework for preparing teachers for classrooms that are inclusive of all students. En N. Florian y N. Pantić (Eds.), Teacher education for the changing demographics of schooling. Issues for Research and Practice (pp. 132-148). Switzerland: Springer International Publishing AG.

Wegerif, R., Postlethwaite, K., Skinner, N., Mansour, N., Morgan, A. y Hetherington, L. (2013). Dialogic Science Education for Diversity. En A. Mansour y R. Wegerif(Eds.), Science Education for Diversity. Theory and Practice (pp. 3-22). Dordrecht: Springer.

Wenger, E. (2002). Comunidades de Práctica: aprendizaje, significado e identidad. España: Paidós.

\section{Notas}

[1] Sistema Nacional de Medición de la Calidad Educativa [SIMCE], que evalúa de forma censal y estandarizada los logros de aprendizaje en las asignaturas de Lenguaje y Comunicación; Matemática; Ciencias Naturales; Historia, Geografía y Ciencias Sociales e Inglés. Las pruebas del SIMCE se aplican a las personas estudiantes de $2^{\circ}, 4^{\circ}, 6^{\circ}, 8^{\circ}$ básico, y II medio (Fuente: https://www.agenciaeducacion.cl/evaluaciones/que-es-el-simce/).

\section{Financiamiento}

Fuente: Agencia Nacional de Investigación y Desarrollo / Programa de Becas / Beca de Doctorado en el Extranjero

No de contrato: BECAS CHILE 72200341 y 72210436 / Beca de Doctorado Nacional 21212101

\section{INFORMACIÓN ADICIONAL}

Cómo citar: Moreno Díaz, K.C., Soto González, R.I. y Oyarzún Maldonado, C.J. (2021). Procesos argumentativos que conforman las reflexiones del profesorado chileno sobre sus experiencias de colaboración en el marco del Diseño Universal para el Aprendizaje. Revista Educación, 45(2). Recuperado de http://doi .org/10.15517/revedu.v45i1.43500 